\title{
Numerical investigation of microbial quorum sensing under various flow conditions
}

\author{
Heewon Jung ${ }^{\text {Corresp., } 1}$, Christof D Meile ${ }^{1}$ \\ 1 University of Georgia, Athens, United States \\ Corresponding Author: Heewon Jung \\ Email address: heewon.jung@uga.edu
}

Microorganisms efficiently coordinate phenotype expressions through a decision-making process known as quorum sensing (QS). We investigated QS amongst distinct, spatially distributed microbial aggregates under various flow conditions using a process-driven numerical model. Model simulations assess the conditions suitable for QS induction and quantify the importance of advective transport of signaling molecules. In addition, advection dilutes signaling molecules so that faster flow conditions require higher microbial densities, faster signal production rates, or higher sensitivities to signaling molecules to induce QS. However, autoinduction of signal production can substantially increase the transport distance of signaling molecules in both upstream and downstream directions. We present empirical approximations to the solutions of the advection-diffusionreaction equation that describe the concentration profiles of signaling molecules for a wide range of flow and reaction rates. These empirical relationships, which predict the distribution of dissolved solutes along pore channels, allow to quantitatively estimate the effective communication distances amongst multiple microbial aggregates without further numerical simulations. 
1

2 Numerical investigation of microbial quorum sensing under 3 various flow conditions

4

5

6 Heewon Jung ${ }^{1}$, Christof Meile ${ }^{1}$

7

$8{ }^{1}$ Department of Marine Sciences, University of Georgia, Athens, GA, USA

9

10

11 Corresponding Author:

12 Heewon Jung ${ }^{1}$

13325 Sanford Dr., Athens, GA, 30602, USA

14 Email address: heewon.jung@uga.edu

15

16 
18

19

20

21

22

23

24

25

26

27

28

29

30

31

32

33

34

35

36

37

38

39

40

41

42

43

44

45

46

47

48

49

50

51

52

53

54

55

56

57

58

59

60

61

62

63

\section{Abstract}

Microorganisms efficiently coordinate phenotype expressions through a decision-making process known as quorum sensing (QS). We investigated QS amongst distinct, spatially distributed microbial aggregates under various flow conditions using a process-driven numerical model. Model simulations assess the conditions suitable for QS induction and quantify the importance of advective transport of signaling molecules. In addition, advection dilutes signaling molecules so that faster flow conditions require higher microbial densities, faster signal production rates, or higher sensitivities to signaling molecules to induce QS. However, autoinduction of signal production can substantially increase the transport distance of signaling molecules in both upstream and downstream directions. We present empirical approximations to the solutions of the advection-diffusion-reaction equation that describe the concentration profiles of signaling molecules for a wide range of flow and reaction rates. These empirical relationships, which predict the distribution of dissolved solutes along pore channels, allow to quantitatively estimate the effective communication distances amongst multiple microbial aggregates without further numerical simulations.

\section{Introduction}

Microorganisms preferentially reside on solid surfaces, which often leads to a closer proximity of neighboring cells than when in a planktonic form (Donné and Dewilde 2015). At elevated cell densities, microorganisms need to efficiently coordinate the expression of energetically expensive phenotypes, such as biofilm development, exoenzyme production, and microbial dispersal. Efficiency is achieved by producing and detecting relatively cheap signaling molecules which regulate the phenotype expression only when a sufficient signal concentration has been reached (Miller and Bassler 2001). This microbial decision-making process called "quorum sensing (QS)" was originally understood as a cell-to-cell communication to identify conspecific population density and accomplish cooperative behaviors (Fuqua et al. 1994). However, a number of studies have indicated that QS is not necessarily a social trait (Redfield 2002; Carnes et al. 2010) and depends not only on the population but also on the spatial distribution of microbial cells (Alberghini et al. 2009; Gao et al. 2016). These observations led to an alternative QS concept in which QS depends strictly on the local concentration of signaling molecules (Hense et al. 2007; Hense and Schuster 2015). This suggests that, to understand QS processes, an integrative approach is required analyzing a multitude of factors including microbial density (Fuqua et al. 1994), production and decay kinetics (Lee et al. 2002; Fekete et al. 2010), and transport of signaling molecules through advection and diffusion (Redfield 2002), as well as the spatial distribution of microorganisms (Alberghini et al. 2009). Thus, spatial constraints and responses may be as important as other biological considerations for the evolution and maintenance of QS. This idea is known to be true in biofilms where cooperative strategies are able to evolve if cooperators are spatially aggregated (Xavier and Foster 2007).

Individual microbial cells synthesize and release signaling molecules at a basal rate. At low population densities, the concentration of signaling molecules remains low as it degrades both biotically and abiotically (Lee et al. 2002; Yates et al. 2002). At a sufficiently high microbial population density, however, the extracellular concentration of signaling molecules reaches a threshold concentration that activates gene and phenotypes expression (Hense and Schuster 2015). When QS regulates the production of costly public goods, this balances production cost and the overall benefit (Pai et al. 2012; Heilmann et al. 2015; Schluter et al. 2016), while under nutrient limited conditions, QS can regulate microbial dispersal (e.g. Solano et al. 2014; Boyle et 
64 al. 2015), improving chances of survival. QS induction also often upregulates genes controlling 65 production of signaling molecules resulting in enhanced signal production (Ward et al. 2001;

66 Fekete et al. 2010; Pérez-Velázquez et al. 2015). Such autoinduction has been thought to confer

67 evolutionary stability and fitness advantages (Brandman et al. 2005; Mitrophanov et al. 2010; Gao and Stock 2018), but its effects on neighboring microbial aggregates and evolutionary benefits in a spatial context have not be fully understood.

QS induction is affected by mass transport characteristics controlling the spatial distribution of signaling molecules. In a confined space, even a single microbial cell can be QS induced if the signaling molecules accumulate to sufficiently high concentration (Carnes et al. 2010). However, higher population densities are required for QS induction in a large open space because the signaling molecules are diluted due to diffusive loss to the surrounding medium (Alberghini et al. 2009; Trovato et al. 2014). Advection may dilute the signaling molecules more effectively than diffusion and repress QS induction. Experimental observations have shown that fast advective flows increase the amount of biomass required for QS induction (Kirisits et al. 2007) and repress QS dependent gene expression (Meyer et al. 2012). Under slower flow conditions, bacteria trapped in a 3D permeable flow cell show more QS dependent gene expressions (Connell et al. 2010). QS induction can be promoted if strong advection is decoupled by heterogeneous pore geometry (e.g. dead-end pores), allowing signaling molecules to accumulate (Kim et al. 2016; Ribbe and Maier, 2016).

The signaling molecules transported either via advection or diffusion can induce QS in neighboring cells (Frederick et al. 2010; Pérez-Velázquez et al. 2016). Because the signal concentration decreases with distance from its source, cells should be located close to each other in order to send and receive enough signaling molecules to and from their neighbors (Hense et al. 2007; Matur et al. 2015). The distance between two QS induced microbial cells or aggregates is referred to as the "calling distance" and has been reported to be $5-78 \mu \mathrm{m}$ between individual cells (Gantner et al. 2006) and $\sim 180 \mu \mathrm{m}$ between microbial aggregates (Darch et al. 2018). However, the dependence of QS processes on advection and diffusion suggests that transport regimes affect calling distances, highlighting the importance of relative positioning of microorganisms coupled with the mass transport characteristics of a habitat.

Here, we evaluate the effect of combined diffusive and advective transport on QS processes in environmentally relevant conditions using a reactive transport modeling approach. The advection-diffusion-reaction equation was nondimensionalized to capture the characteristic properties of QS systems (i.e. effects of the rate of signaling molecule production, cell density, mass transport, and spatial distribution of microbial aggregates) and used to formulate empirical expressions describing concentration profiles of signaling molecules under various flow conditions. Using these relationships, we evaluate calling distances and threshold biochemical conditions for QS induction of a single microbial aggregate under various flow conditions. Then, we investigate QS interactions between heterogeneously distributed microbial aggregates. Finally, we demonstrate the importance of autoinduction for coordinated microbial behaviors in advection-dominated environments. This study quantifies the effect of flow velocities, autoinduction, and relative position of microbial aggregates to calling distances in a $2 \mathrm{D}$ flow channel.

\section{Materials \& Methods}

We used the Lattice Boltzmann (LB) method to implement a numerical model for the transport of signaling molecules due to diffusion and advection. The LB method is a mesoscopic 
110

111

112

113

114

115

116

117

118

119

120

121

122

123

124

125

126

127

128

129

130

131

132

133

134

135

136

137

138

139

approach solving the Boltzmann equation across a defined set of particles which recovers the macroscopic Navier-Stokes equation (NSE) and advection-diffusion-reaction equation (ADRE) (Tang et al. 2013; Krüger et al. 2017). First, we obtained the flow field by solving the particle distribution function $f$ :

$$
f_{i}\left(\mathbf{r}+\boldsymbol{c}_{i} \Delta t, t+\Delta t\right)=f_{i}(\mathbf{r}, t)+\frac{\Delta t}{\tau}\left(f_{i}^{e q}(\mathbf{r}, t)-f_{i}(\mathbf{r}, t)\right)
$$

where particles $f_{i}(\mathbf{r}, \mathrm{t})$ travel in the direction $i$ with the lattice velocity $\boldsymbol{c}_{i}\left(\boldsymbol{c}_{0}=(0,0), \boldsymbol{c}_{1}=(1,0), \boldsymbol{c}_{2}\right.$ $\left.=(0,1), \boldsymbol{c}_{3}=(-1,0), \boldsymbol{c}_{4}=(0,-1), \boldsymbol{c}_{5}=(1,1), \boldsymbol{c}_{6}=(-1,1), \boldsymbol{c}_{7}=(-1,-1), \boldsymbol{c}_{8}=(1,-1)\right)$ to a new position $\mathbf{r}+\boldsymbol{c}_{i} \Delta \mathrm{t}$ after a time step $\Delta \mathrm{t}$. The relaxation time $(\tau)$ was described by the commonly used Bhatnagar-Gross-Krook collision operator (Bhatnagar et al. 1954) and the D2Q9 lattice with the corresponding equilibrium distribution function:

$$
f_{i}^{e q}(\mathbf{r}, t)=\omega_{i} \rho\left(1+\frac{\mathbf{u} \cdot \mathbf{c}_{i}}{c_{s}^{2}}+\frac{\left(\mathbf{u} \cdot \mathbf{c}_{i}\right)^{2}}{2 c_{s}^{4}}-\frac{\mathbf{u} \cdot \mathbf{u}}{2 c_{s}^{2}}\right)
$$

where $\omega_{i}$ are lattice weights $\left(\omega_{0}=4 / 9, \omega_{1-4}=1 / 9, \omega_{5-8}=1 / 36\right), c_{s}$ is a lattice dependent constant (here, $c_{s}^{2}=1 / 3$ ), and $\mathbf{u}$ is the macroscopic flow velocity. The moments of the discretized mesoscopic particles retrieve the macroscopic density $\left(\rho=\Sigma f_{i}\right)$ and momentum $\left(\rho \mathbf{u}=\Sigma \boldsymbol{c}_{i} f_{i}\right)$. The Chapman-Enskog expansion showed that this LB approach recovers the incompressible NSE with the viscosity $v=c_{s}^{2}\left(\tau-\frac{\Delta t}{2}\right)$ (Krüger et al. 2017). Once the flow field was obtained, we simulated solute transport with a particle distribution function $g$, using the regularized LB algorithm (RLB) for numerical accuracy (Latt and Chopard 2006; Latt 2007) and the D2Q5 lattice for numerical efficiency (Li et al. 2017):

$$
\mathrm{g}_{i}\left(\mathbf{r}+\boldsymbol{c}_{i} \Delta t, t+\Delta t\right)=\mathrm{g}_{i}^{e q}(\mathbf{r}, t)+\left(1-\frac{\Delta t}{\tau}\right) \frac{\omega_{i}}{2 c_{s}^{4}} \boldsymbol{Q}_{i}: \boldsymbol{\Pi}_{i}^{n e q}+\Omega_{i}^{R X N}(\mathbf{r}, t)
$$

where $\boldsymbol{c}_{i}$ are the lattice velocities $\left(\boldsymbol{c}_{0}=(0,0), \boldsymbol{c}_{1}=(1,0), \boldsymbol{c}_{2}=(0,1), \boldsymbol{c}_{3}=(-1,0), \boldsymbol{c}_{4}=(0,-1), \boldsymbol{c}_{5}=\right.$ $(1,1))$ corresponding to the lattice weights $\omega_{i}\left(\omega_{0}=1 / 3, \omega_{1-4}=1 / 6\right)$, and $\boldsymbol{Q}_{i}: \boldsymbol{\Pi}_{i}^{\text {neq }}$ is the tensor contraction between the two tensors $\boldsymbol{Q}_{i}=\boldsymbol{c}_{i} \cdot \boldsymbol{c}_{i}^{T}-c_{s}^{2} \boldsymbol{I}$ and $\boldsymbol{\Pi}_{i}^{\text {neq }}=\sum_{j} \boldsymbol{c}_{i} \cdot \boldsymbol{c}_{i}^{T}\left(\mathrm{~g}_{j}(\mathbf{r}, t)-\mathrm{g}_{j}^{e q}(\mathbf{r}, t)\right)$. The reaction term in the Eq. 3 describes the production of signaling molecules:

$$
\Omega_{i}^{R X N}(\mathbf{r}, t)=\Delta t \omega_{i}(1+F H[\hat{A}-\hat{\theta}]) \hat{k} \hat{B}
$$

where $F$ represents a multiplication factor which was set to either 10 to reflect the magnitude of autoinduced signal production (Fekete et al. 2010), $\hat{A}$ is a concentration of signaling molecules, $\hat{\theta}$ is the QS induction threshold, $\hat{k}$ is the basal production rate constant of signaling molecules, and $\hat{B}$ is the microbial density. QS induction often displays a switch-like behavior (Fujimoto and Sawai 2013; Heilmann et al. 2015; Hense and Schuster 2015), which is represented in the model by a step function with a higher signal production rate above the threshold concentration of signaling molecule:

$$
H[\hat{A}-\hat{\theta}]=\left\{\begin{array}{l}
1,(\hat{A} \geq \hat{\theta}), \\
0,(\hat{A}<\hat{\theta}) .
\end{array}\right.
$$

With the imposed flow field from Eq. 1, the LB transport solver (Eq. 3) recovers the following ADRE:

$$
\frac{\partial \hat{A}}{\partial \hat{t}}+\hat{\mathbf{u}} \cdot \hat{\nabla} \hat{A}=\hat{D} \hat{\nabla}^{2} \hat{A}+(1+F H[\hat{A}-\hat{\theta}]) \hat{k} \hat{B}
$$


140 with the molecular diffusivity $\hat{D}=c_{s}^{2}\left(\tau-\frac{\Delta t}{2}\right)$. Note that we are ignoring the breakdown of signaling

141

142

143

144

145

146

147

148

149

150

151

152

153

154

155

156

157

158

159

160

161

162

163

164

165

166

167

168

169

170

171

172

173

174

175

176

177

178

179

180

molecules (Lee et al. 2002), limiting us to settings where production and transport are the dominant processes.

To describe the characteristic properties of a microbial system across various flow and reaction conditions, Eq. 6 was recast by introducing dimensionless quantities $A=\frac{\hat{A}}{\hat{\theta}}, t=\frac{\hat{D} \hat{t}}{\hat{l}^{2}}, \nabla=\hat{\nabla} \hat{l}$, $B=\frac{\hat{B}}{\hat{B}_{\theta}}, \mathbf{u}=\hat{\hat{u}}$, where $\hat{l}$ is a characteristic length (i.e. the width of the flow channel), $\hat{U}$ is a characteristic fluid velocity (here, the average pore fluid velocity), and $\hat{B}_{\theta}$ is a threshold biomass density required for $\mathrm{QS}$ induction, resulting in:

$$
\frac{\partial A}{\partial t}+P e \mathbf{u} \cdot \nabla A=\nabla^{2} A+D a
$$

This nondimensionalized ADRE is fully characterized by the Péclet number, expressing the magnitude of advective flow relative to diffusion $\left(P e=\frac{\hat{U} \hat{i}}{\hat{D}}\right)$, and the diffusive Damköhler number, comparing reaction to diffusion $\left(D a=\frac{k^{\prime} B \hat{l}^{2}}{\hat{\theta} \hat{D}}\right.$; where $\left.k^{\prime}=(1+F H[\hat{A}-\hat{\theta}]) \hat{k} \hat{B}_{\theta}\right)$. A system with high $D a$ - either due to high $k^{\prime}$ (i.e. fast signal production), high $B$ (i.e. high microbial density), or low $\hat{\theta}$ (i.e. high sensitivity to signaling molecules) - is more likely to be QS induced.

An important property of Eq. 7 is that its solution linearly scales in $D a$ (Lin et al. 2020). For example, if $D a$ is increased 2-fold at a fixed $P e$ condition, the concentrations of signaling molecule are doubled. This linearity allows to calculate the concentration distribution of signaling molecules for any $D a$ from a single simulation result with an arbitrary $D a$ at a given $P e$. However, this simple approach cannot be applied to the flow conditions because the solution is not linear in $P e$. Therefore, multiple numerical simulations were carried out with $24 \mathrm{Pe}$ conditions $(P e \in\{0.5,0.6,0.7,0.8,0.9,1,1.5,2,2.5,3,3.5,4,4.5,5,5.5,6,6.5,7,7.5,8,8.5$, $9,9.5,10\}$ ) while $D a$ was fixed at 5. For the 2D simulations in a straight channel (Fig. 1), the flow field was established by imposing pressures at in- and outlet and no flow conditions at the top and bottom boundaries, resulting in a flow from left to right. Fixed concentration $\left(\left.A\right|_{\text {left }}\right.$ boundary, $\mathrm{x}=0=0)$ and no-gradient $\left(\partial A /\left.\partial x\right|_{\text {right boundary, } \mathrm{x}=4}=0\right)$ boundary conditions were imposed at the inlet and outlet boundaries, with no-flux at the top and bottom boundaries, respectively. All simulations were run to steady state.

Simulations were conducted for a 2D flow channel of non-dimensional length of 4 and a width of 2 , discretized with $2000 \times 1000$ grid elements. The flow field (Eq. 1) was generated by imposing fixed pressures at inlet $(x=0)$ and outlet $(x=4)$ with no flow boundaries in both normal and tangential direction at the bottom $(y=0)$ and top $(y=2)$ of the domain resulting in parabolic Poiseuille flows. Simulations were carried out under low Mach numbers $\left(M a=\mathbf{u} / c_{s} \ll\right.$ 1) to ensure incompressible flow conditions (Krüger et al. 2017).

\section{Results and discussion}

\section{QS processes of a single microbial aggregate}

The effect of various flow conditions on the distribution of signaling molecules $(A)$ produced from a single microbial aggregate assumed a source constrained to a single grid cell located at $x$ $=1$ was investigated under various $P e$ conditions $(0.5 \leq P e \leq 10)$ while $D a$ was fixed at 5 (Fig. 1). The environmentally relevant range of $P e$ was chosen (Battiato et al. 2009, 2011) while $D a$ is arbitrary because of the linearity of Eq. 7 in $D a$. The QS induction enhancing the signal production rate was not considered. 
181

182

183

184

185

186

187

188

189

190

191

192

193

194

195

196

197

198

199

200

201

202

203

204

205

206

207

208

209

210

211

212

213

214

215

216

217

218

219

220

221

222

223

224

The signal concentration fields developed under various advective flows show maximum concentrations $\left(A_{\max }=A(x=1)\right)$ decreasing with increasing $P e$ (i.e. faster advective flow): $A_{\max }$ decreased from $1.68(P e=1)$ to $1.35(P e=5)$ and $1.21(P e=10)$. However, $A_{\max }$ of all of the simulations with $D a=5$ exceeded 1(i.e. $\hat{A} \geq \hat{\theta})$, indicating the potential for QS induction. The threshold $D a$ for QS induction $\left(D a_{\theta}\right)$, where $A_{\max }=1$, can easily be computed using the linearity of the nondimensionalized ADRE in $D a$ (Eq. 7). For example, $D a_{\theta}$ at $P e=1$ was calculated by dividing $D a=5$ by its corresponding $A_{\max }=1.68$ which resulted in $D a_{\theta}=2.98$. Thus, at $P e=1$, conditions for which $D a \geq 2.98$ lead to or exceed the concentration of signaling molecules needed for QS induction. Fig. 2 shows the calculated $D a_{\theta}$ for each simulated $P e$ condition.

The regression analysis revealed that the simulated $D a_{\theta}$ for QS induction varies as a function of $P e$ following the power law:

$$
D a_{\theta}=1.3812 P e^{0.2626}+1.592
$$

The increasing $D a_{\theta}$ along with the increasing $P e$ indicates higher $D a$ (i.e. higher microbial density $(B)$, higher signal production rate constant $\left(k^{\prime}\right)$, or lower QS induction threshold $\left.(\hat{\theta})\right)$ is required for QS induction under higher $P e$. This result corresponds to the observed repressed QS induction under the presence of advection (Vaughan et al. 2010; Meyer et al. 2012; Kim et al. 2016) and matches the pattern of biomass required for QS under varying flow conditons (Kirisits et al. 2007). Eq. 8 was further evaluated by applying the experimentally measured QS parameters of Pseudomonas putida $\left(\hat{k}=2.3 \times 10^{-10} \mathrm{nmol} / \mathrm{cell} / \mathrm{h}\right.$, and $\hat{\theta}=70 \mathrm{nmol} / \mathrm{L}$ (Fekete et al. 2010$\left.)\right)$ in a flow system where $\hat{l}=1 \mathrm{~cm}$ and $\hat{D}=3.0 \times 10^{-10} \mathrm{~m}^{2} / \mathrm{s}$ (Dilanji et al. 2012). Our results show $\hat{B}_{\theta}$ of 9.77, 12.2 , and $13.5 \times 10^{6}$ cells/mL at $P e=1,5$, and 10 , respectively. If Eq. 8 is extrapolated to diffusion only transport condition $\left(P e=0, D a_{\theta}=1.592\right), \hat{B}_{\theta}$ is estimated as $5.23 \times 10^{6} \mathrm{cell} / \mathrm{s} / \mathrm{mL}$ which largely agree with the experimental observation of $2.69 \sim 6.23 \times 10^{6}$ cells $/ \mathrm{mL}$ where signal concentration starts to show a strong spike (Table S1 in Fekete et al. 2010).

In addition to reducing $A_{\max }$, advection also influenced the spatial distribution of signaling molecules. We define the "transport distance" $(d)$ as the distance between the point of production $\left(x_{0}\right)$ and the point $\left(x_{1}\right)$ where the signal concentration reaches a certain value $A^{*}$ (i.e. $d=\left|x_{0}-x_{1}\right|$ ), distinguishing it from the "calling distance" between two QS induced microbial cells or aggregates. If the signal transport occurred only through diffusion, transport distances would be isotropic (Alberghini et al. 2009). However, advection resulted in anisotropic concentration distribution where upstream transport distances $\left(d_{u p}\right)$ are much shorter than the downstream distances $\left(d_{d n}\right)$. Moreover, fast advective flows (i.e. high $P e$ ) reduced overall transport distances which are illustrated in Fig. 1a-c as the shrinking areas covered by contour lines. For example, the (nondimensional) transport distances to the location where $A=0.1$ are $d_{u p}=0.08$ and $d_{d n}=$ 0.62 at $P e=1$ and $D a=5$ (Fig. 1a). These values decrease to $d_{u p}=0.033$ and $d_{d n}=0.27$ at $P e=5$ (Fig. 1b) and to $d_{u p}=0.023$ and $d_{d n}=0.19$ at $P e=10$ (Fig. 1c).

\section{Empirical approximation of concentration profiles}

Obtaining transport distances for different $P e$ conditions requires running numerical simulations for each of the corresponding $P e$. However, this may be avoided if we can express the concentration profiles as a function of $\mathrm{Pe}$. For this purpose, parametric regression analysis was applied to the numerically obtained concentration profiles along the bottom of the flow channel (Fig. 3).

Several parametric regression models (linear, power, exponential, and polynomial models) were tested to the upstream $\left(A_{u p}(x) ; 0 \leq x \leq 1\right)$ and downstream $\left(A_{d n}(x) ; 1<x \leq 4\right)$ signal 
225

226

227

228

229

230

231

232

233

234

235

236

237

238

239

240

241

242

243

244

245

246

247

248

249

250

251

252

253

254

255

256

257

concentration profiles. Among the tested regression models, the exponential (Eq. 9) and powerlaw models (Eq. 10) provided the best fit for log-transformed upstream and downstream signal concentration profiles, respectively. In the regression analysis of upstream profiles, only the locations where $A(x)>0.001$ were used to improve the fitting quality and the signal concentration at $x=1$ was fixed as 1 . The additional regression analysis was then carried out for the coefficients ( $a, b$, and $c)$ obtained from simulated profiles at $24 P e$ conditions to construct a relationship between the coefficients and $P e$ (Fig. 3b-d). The exponential and power-law models provided the best fit for the upstream (Eqs. 11-13) and downstream coefficients (Eqs. 14-16), respectively:

$$
\begin{gathered}
\left.A_{u p}(x)\right|_{x \leq 1}=\exp \left(a_{u p}\left(x^{b} u p-x^{c} u p\right)\right) \\
\left.A_{d n}(x)\right|_{x>1}=\exp \left(a_{d n} \ln (x)^{b} d n+c_{d n}\right)
\end{gathered}
$$

Eqs. 9 and 10 can be used as approximations of the concentration profiles along a pore channel without running simulations for various $P e$ conditions, with the microbial aggregate located at $x=1$. Due to the linearity in $D a$, the concentration profiles at different $D a$ conditions can be calculated simply by multiplying $D a / D a_{\theta}$ to Eqs. 9 and 10 , so that

$$
A(x)=\frac{D a}{D a_{\theta}}\left(A_{u p}(x)+A_{d n}(x)\right)
$$

These analytical expressions are applicable not only to QS but also to other chemical processes subject to zero-order production reactions (e.g. Bezemer et al. 2000; Tang et al. 2015). The equations become less accurate at low $\mathrm{Pe}$ as under low flow conditions, the estimates from Eq. 17 in a flow channel with a small width (i.e. low $\hat{l}$ and $P e$ ) could underestimate the actual concentration because the confined channel width would push the produced chemical further upstream and downstream.

\section{The effect of QS induced signal production on transport distances}

QS often involves autoinduction which substantially increases signal production rates. The effect of autoinduction on transport distances was investigated by using Eq. 17 for the conditions without $\left(F=0 ; D a=D a_{\theta}\right)$ and with $\left(F=10 ; D a=11 D a_{\theta}\right)$ enhanced signal production. The transport distances from a single microbial aggregate under various $P e$ were then calculated using Eq. 17 for the location $x$.

Fig. 4 shows the transport distances without (Fig. 4a) and with (Fig. 4b) the enhanced signal production at $P e=1,5$, and 10 . The concentration ratios $\left(0.1 \leq A / A_{\max } \leq 0.9\right)$ were used instead of absolute concentrations to generalize transport distances for various $D a$ conditions. For example, the transport distance $\left(d_{A}\right)$ for $A / A_{\max }=0.5$ indicates that $A\left(x_{0}+d_{A}\right)=0.5$ if $D a=D a_{\theta}$ while $A\left(x_{0}+d_{A}\right)=0.05$ when $D a=0.1 D a_{\theta}$. The consequence of the enhanced signal production was the significant increase of $d_{u p}$ and $d_{d n}$. Without the enhanced signal production, $d_{u p}$ and $d_{d n}$

Peer] reviewing PDF | (2020:04:47932:1:0:NEW 11 Jul 2020) 
258 for $A / A_{\max }=0.4$ at $P e=1$ were estimated as 0.021 and 0.024 , respectively (Fig. 4a). These values 259 increased to $d_{u p}=0.1$ and $d_{d n}=1.28$ with the enhanced signal production (Fig. $4 \mathrm{~b}$ ). The

260 downstream transport distance of 1.28 is translated into $6.4 \mathrm{~mm}$ in a flow channel with $\hat{l}=1 \mathrm{~cm}$.

261 This result is much longer than the generally observed ranges of calling distances (Whiteley et al.

262

263

264

265

266

267

268

269

270

271

272

273

274

275

276

277

278

279

280

281

282

283

284

285

286

287

288

289

290

291

292

293

294

295

296

297

298

299 2017). However, we emphasize again that the transport distance merely indicates the distance of signaling molecules transported from a source location while the calling distance involves QS induced microbial cells or aggregates.

\section{QS induction between spatially distributed multiple microbial aggregates}

QS processes of multiple aggregates were investigated by constructing the concentration profiles using Eq. 18. Concentration fields of signaling molecules with multiple microbial aggregates can be calculated as the superposition of the concentration profile produced by each individual aggregate:

$$
A(x)=\sum_{i=1}^{n} \frac{D a_{i}}{D a_{\theta}}\left(A_{u p}\left(x+d_{i 0}\right)+A_{d n}\left(x+d_{i 0}\right)\right)
$$

where $n$ is the number of aggregates, $d_{i 0}$ is the distance between $x_{i}$ and $x_{0}\left(d_{i 0}=x_{i}-x_{0}\right), x_{i}$ is the location of $i$ th aggregate, $x_{0}$ is the reference location $\left(x_{0}=1\right), D a_{\mathrm{i}}$ is the $D a$ calculated only with the density of $i$ th microbial aggregate (i.e. microscopic $D a$ ), and $A_{u p}$ and $A_{d n}$ are Eqs. 9 and 10, respectively. Here, an example system with macroscopic $D a\left(D a_{\mathrm{T}}=\Sigma D a_{\mathrm{i}}\right)=3.2 D a_{\theta}$ consist of four aggregates $\left(\mathbb{A}_{1-4}\right)$ located at $x_{1}=0.4, x_{2}=1, x_{3}=1.096$, and $x_{4}=1.7$ with the evenly distributed microscopic $D a_{\text {i }}$ (i.e. $D a_{1}=D a_{2}=D a_{3}=D a_{4}=0.8 D a_{\theta}$ ) was tested. In using Eq. 18, the profile was first constructed for $D a_{i}=D a^{*}$ that does not consider autoinduction $(F=0)$. Then, if there is an aggregate with $A\left(x_{\mathrm{i}}\right) \geq 1$, the profile was recalculated with updated $D a_{\mathrm{i}}=$ $(1+F) \times D a^{*}$ until all $D a_{\mathrm{i}}$ with $A \geq 1$ were updated.

The signal concentration profile produced by four aggregates without the enhanced signal production $(F=0)$ illustrates the crucial importance of relative positioning of microbial aggregates for QS induction with respect not only to each aggregate but also to the flow direction (Fig. 5a). The microscopic $D a_{\mathrm{i}}$ was set such that the maximum concentration produced by a single aggregate was 0.8 , as observed at the most upstream location $\left(\mathbb{A}_{1}\right.$ at $\left.x_{1}=0.4\right)$. But due to transport, the local concentration at $\mathbb{A}_{2}$ reached 0.879 , receiving $A$ of 0.048 and 0.031 from $\mathbb{A}_{1}$ and $\mathbb{A}_{3}$, respectively. $\mathbb{A}_{3}$ received slightly less signaling molecules from $\mathbb{A}_{1}(A=0.044)$ due to the longer distance of $\mathbb{A}_{3}$ than $\mathbb{A}_{2}$ from $\mathbb{A}_{1}$. However, $\mathbb{A}_{2}$ provided much more signaling molecules ( $A$ $=0.157)$ to $\mathbb{A}_{3}$ than was provided by $\mathbb{A}_{3}$ because of advective flows favoring downstream transport of signaling molecules (Figs. 2 and 4). As a consequence, $\mathbb{A}_{3}$ exceeded the QS threshold $\left(A\left(x_{3}\right)=0.044\right.$ from $\mathbb{A}_{1}+0.157$ from $\mathbb{A}_{2}+0.8$ from $\mathbb{A}_{3}+0$ from $\left.\mathbb{A}_{4}=1.001>1\right)$ while the upstream located $\mathbb{A}_{2}$ did not. The $\mathrm{QS}$ induction of $\mathbb{A}_{3}$ demonstrates the importance of transport distances. QS induction was achieved because of the upstream aggregates located within the transport distance of 0.696 . However, the calling distance would have been estimated as the length of a grid voxel $(0.002)$ because only $\mathbb{A}_{3}$ was $Q S$ induced. Therefore, considering only the calling distance could lead to the wrong conclusion that the local $D a$ condition at $\mathbb{A}_{3}$ (i.e. $D a_{3}=$ $\left.0.8 D a_{\theta}\right)$ is a sufficient condition for QS induction. Although $\mathbb{A}_{4}$ did not reach the QS induction threshold, it received $A$ from all the other aggregates resulting in a concentration $\left(A\left(x_{4}\right)=0.029+\right.$ $0.044+0.048+0.8=0.921)$ that was higher than at $\mathbb{A}_{2}$ despite the longest separation distance from other aggregates. 
Accounting for QS induction $(F=10)$ increased the transport distances and hence induced other aggregates (Fig. 5b). With the same spatial distribution, QS-induced $\mathbb{A}_{3}$ produced signaling molecules much more and faster (i.e. $k^{\prime}=11 \hat{k}$ and $D a_{3}=8.8 D a_{\theta}$ ) and provided more signaling molecules to $\mathbb{A}_{2}$. As a result, $A\left(x_{2}\right)$ exceeded the QS threshold $(0.048+0.8+0.335+0=1.183)$. The QS induction of $\mathbb{A}_{2}$ and $\mathbb{A}_{3}$ resulted in the final signal concentrations of $A\left(x_{2}\right)=9.183(=$ $0.048+8.8+0.035+0)$ and $A\left(x_{3}\right)=10.569(=0.044+1.725+8.8+0)$. While $\mathbb{A}_{4}$ still did not contribute signaling molecules to any of upstream aggregates, enhanced contribution from $\mathbb{A}_{2}$ and $\mathbb{A}_{3}$ QS induced $\mathbb{A}_{4}, A\left(x_{4}\right)=9.839(=0.029+0.48+0.53+8.8)$. Despite increased transport distances by QS induction, $\mathbb{A}_{1}$ was still too far away from the other aggregates thus the signal concentration at $\mathbb{A}_{1}$ remained unchanged $A\left(x_{1}\right)=0.8$. As a result of the QS induction of $\mathbb{A}_{2-4}, D a_{\mathrm{T}}$ had increased from the initial $3.2 D a_{\theta}\left(=0.8 D a_{\theta} \times 4\right)$ to $27.2 D a_{\theta}\left(=0.8 D a_{\theta}+3 \times 11 \times 0.8 D a_{\theta}\right)$.

This example illustrates the importance of enhanced signal production on the spatial propagation of QS induction. While only $\mathbb{A}_{3}$ experienced signaling molecule levels that could induce QS when all the aggregates produce signaling molecules at the basal production rate, the enhanced signal production of $\mathbb{A}_{3}$ when considering induced production $(F=10)$ provided more signaling molecules to its adjacent microbial aggregates and resulted in the QS induction of neighboring aggregates, $\mathbb{A}_{2}$ and $\mathbb{A}_{4}$. It may be counterintuitive that the upstream-located $\mathbb{A}_{2}$ was also QS-induced by the contribution from $\mathbb{A}_{3}$ despite the contracted upstream transport distances under the presence of advective flows. This result shows that the enhanced signal production can overcome the influence of advection and promote QS induction, and provide a way to provoke upstream microbial aggregates, for example, to slow down the substrate consumption to ensure efficient resource utilization in crowded environments (An et al. 2014).

\section{Conclusions and perspectives}

This study has demonstrated that advection and the enhanced signal production can determine the spatial extent of QS induction. Reactive transport simulation results reveal that fast flow conditions dilute signaling molecules and thus higher $D a_{\theta}$ (i.e. faster signal production rate, higher microbial density, or lower QS induction threshold concentration) is required for QS induction. Reduced upstream delivery of signaling molecules under advective flow limits propagation of QS; it can be relaxed if autoinduction increases signal production rates. Our study results highlight the importance of relative positioning of microbial aggregates with respect to flow directions and the role of autoinduction to overcome advection for upstream signal propagation.

The simulations focused on the effect of various flow conditions on QS and assumed that microbial aggregates have a negligible impact on flow fields, which is a reasonable approximation for low microbial density conditions. However, it may not hold when large aggregates producing extracellular polymeric substances (EPS) perturb flows substantially. In such a case, estimating signal transport requires fully resolving nonlinear feedback between cell activity and fluid flow (Thullner and Baveye 2008; Carrel et al. 2018; Jung and Meile 2019), including diffusion limitation (Stewart 2003). Finally, accounting for degradation of signaling molecules (Lee et al. 2002; Yates et al. 2002) and increased spreading of signaling molecules in $3 \mathrm{D}$ systems than $2 \mathrm{D}$, would result in shorter transport distances than this study.

Although QS mediated gene expression has been understood as evolutionarily beneficial collective behaviors, long transport distances observed in this study suggests that it may not be always true. The transport of signaling molecules, especially in downstream direction, combined with enhanced signal production, suggests that QS induction can be decoupled from microbial 
346 density. In the above example (Fig. 5b), any microbial cell located where $A>1$ (e.g. $A(x=2.5)=$ 347 1.05) would have been QS-induced, independent of the local cell density. This could lead to 348 detrimental impacts on a microbial population, unless there are other counteracting mechanisms 349 such as differential QS induction sensitivity to signal concentration even in within a clonal 350 population (Darch et al. 2018) or biofilm formation modifying local transport characteristics 351 (Emge et al. 2016). Future investigations should explicitly examine the evolutionary 352 consequences of QS strategies in spatially heterogeneous environments under advective353 diffusion-reaction dynamics.

354

\section{Acknowledgement}

We thank Chris Kempes for constructive comments, and Eleonora Secchi and 2 anonymous reviewers for feedback that helped improve on the manuscript. 


\section{References}

359 Alberghini S, Polone E, Corich V, et al (2009) Consequences of relative cellular positioning on

360

361

362

363

364

365

366

367

368

369

370

371

372

373

374

375

376

377

378

379

380

381

382

383

384

385

386

387

388

389

390

391

392

393

394

395

396

397

398

399

400

401

402

403 quorum sensing and bacterial cell-to-cell communication. FEMS Microbiol Lett 292:149161. https://doi.org/10.1111/j.1574-6968.2008.01478.x

An JH, Goo E, Kim H, et al (2014) Bacterial quorum sensing and metabolic slowing in a cooperative population. Proc Natl Acad Sci U S A 111:14912-14917. https://doi.org/10.1073/pnas.1412431111

Battiato I, Tartakovsky DM, Tartakovsky AM, Scheibe TD (2009) On breakdown of macroscopic models of mixing-controlled heterogeneous reactions in porous media. Adv Water Resour 32:1664-1673. https://doi.org/10.1016/j.advwatres.2009.08.008

Battiato I, Tartakovsky DM, Tartakovsky AM, Scheibe TD (2011) Hybrid models of reactive transport in porous and fractured media. Adv Water Resour 34:1140-1150. https://doi.org/10.1016/j.advwatres.2011.01.012

Bezemer JM, Radersma R, Grijpma DW, et al (2000) Zero-order release of lysozyme from poly(ethylene glycol)/poly(butylene terephthalate) matrices. J Control Release 64:179-192. https://doi.org/10.1016/S0168-3659(99)00127-3

Bhatnagar PL, Gross EP, Krook M (1954) A Model for Collision Processes in Gases. I. Small Amplitude Processes in Charged and Neutral One-Component Systems. Phys Rev 94:511525. https://doi.org/10.1103/PhysRev.94.511

Boyle KE, Monaco H, van Ditmarsch D, et al (2015) Integration of Metabolic and Quorum Sensing Signals Governing the Decision to Cooperate in a Bacterial Social Trait. PLoS Comput Biol 11:1-26. https://doi.org/10.1371/journal.pcbi.1004279

Brandman O, Ferrell JE, Li R, Meyer T (2005) Positive feedback loops drive reliable cell decisions. Science 310:496-499

Carnes EC, Lopez DM, Donegan NP, et al (2010) Confinement-induced quorum sensing of individual Staphylococcus aureus bacteria. Nat Chem Biol 6:41-45. https://doi.org/10.1038/nchembio.264

Carrel M, Morales VL, Beltran MA, et al (2018) Biofilms in 3D porous media: Delineating the influence of the pore network geometry, flow and mass transfer on biofilm development. Water Res 134:280-291. https://doi.org/10.1016/j.watres.2018.01.059

Connell JL, Wessel AK, Parsek MR, Ellington AD, Whiteley M, Shear JB (2010) Probing prokaryotic social behaviors with bacterial "lobster traps". MBio 1:1-8. https://doi.org/10.1128/mBio.00202-10.

Darch SE, Simoska O, Fitzpatrick M, et al (2018) Spatial determinants of quorum signaling in a Pseudomonas aeruginosa infection model. Proc Natl Acad Sci U S A 115:4779-4784. https://doi.org/10.1073/pnas.1719317115

Dilanji GE, Langebrake JB, De Leenheer P, Hagen SJ (2012) Quorum activation at a distance: Spatiotemporal patterns of gene regulation from diffusion of an autoinducer signal. $\mathrm{J}$ Am Chem Soc 134:5618-5626. https://doi.org/10.1021/ja211593q

Donné J, Dewilde S (2015) The Challenging World of Biofilm Physiology. In: Advances in Microbial Physiology, 1st edn. Elsevier Ltd., pp 235-292

Emge P, Moeller J, Jang H, et al (2016) Resilience of bacterial quorum sensing against fluid flow. Sci Rep 6:33115. https://doi.org/10.1038/srep33115

Fekete A, Kuttler C, Rothballer M, et al (2010) Dynamic regulation of N-acyl-homoserine lactone production and degradation in Pseudomonas putida IsoF. FEMS Microbiol Ecol 72:22-34. https://doi.org/10.1111/j.1574-6941.2009.00828.x 
404 Fujimoto K, Sawai S (2013) A Design Principle of Group-level Decision Making in Cell

405

406

407

408

409

410

411

412

413

414

415

416

417

418

419

420

421

422

423

424

425

426

427

428

429

430

431

432

433

434

435

436

437

438

439

440

441

442

443

444

445

446

447

448

449

Populations. PLoS Comput Biol 9:. https://doi.org/10.1371/journal.pcbi.1003110

Fuqua WC, Winans SC, Greenberg EP (1994) Quorum sensing in bacteria: The LuxR-LuxI family of cell density- responsive transcriptional regulators. J Bacteriol 176:269-275. https://doi.org/10.1128/jb.176.2.269-275.1994

Gantner S, Schmid M, Dürr C, et al (2006) In situ quantitation of the spatial scale of calling distances and population density-independent $\mathrm{N}$-acylhomoserine lactone-mediated communication by rhizobacteria colonized on plant roots. FEMS Microbiol Ecol 56:188194. https://doi.org/10.1111/j.1574-6941.2005.00037.x

Gao M, Zheng H, Ren Y, et al (2016) A crucial role for spatial distribution in bacterial quorum sensing. Sci Rep 6:1-10. https://doi.org/10.1038/srep34695

Gao R, Stock AM (2018) Overcoming the Cost of Positive Autoregulation by Accelerating the Response with a Coupled Negative Feedback. Cell Rep 24:3061-3071.e6. https://doi.org/10.1016/j.celrep.2018.08.023

Heilmann S, Krishna S, Kerr B (2015) Why do bacteria regulate public goods by quorum sensing?-How the shapes of cost and benefit functions determine the form of optimal regulation. Front Microbiol 6:1-11. https://doi.org/10.3389/fmicb.2015.00767

Hense BA, Kuttler C, Müller J, et al (2007) Does efficiency sensing unify diffusion and quorum sensing? Nat Rev Microbiol 5:230-239. https://doi.org/10.1038/nrmicro1600

Hense BA, Schuster M (2015) Core Principles of Bacterial Autoinducer Systems. Microbiol Mol Biol Rev 79:153-169. https://doi.org/10.1128/mmbr.00024-14

Jung H, Meile C (2019) Upscaling of microbially driven first-order reactions in heterogeneous porous media. J Contam Hydrol 224:103483. https://doi.org/10.1016/j.jconhyd.2019.04.006

Kim MK, Ingremeau F, Zhao A, et al (2016) Local and global consequences of flow on bacterial quorum sensing. Nat Microbiol 1:1-5. https://doi.org/10.1038/nmicrobiol.2015.5

Kirisits MJ, Margolis JJ, Purevdorj-Gage BL, et al (2007) Influence of the hydrodynamic environment on quorum sensing in Pseudomonas aeruginosa biofilms. J Bacteriol 189:8357-8360. https://doi.org/10.1128/JB.01040-07

Krüger T, Kusumaatmaja H, Kuzmin A, et al (2017) The Lattice Boltzmann Method. Springer International Publishing, Cham

Latt J (2007) Hydrodynamic limit of lattice Boltzmann equations. Université de Genève. Thèse. doi:10.13097/archive-ouverte/unige:464 https://archive-ouverte.unige.ch/unige:464

Latt J, Chopard B (2006) Lattice Boltzmann method with regularized pre-collision distribution functions. Math Comput Simul 72:165-168. https://doi.org/10.1016/j.matcom.2006.05.017

Lee SJ, Park SY, Lee JJ, et al (2002) Genes encoding the N-acyl homoserine lactone-degrading enzyme are widespread in many subspecies of Bacillus thuringiensis. Appl Environ Microbiol 68:3919-3924. https://doi.org/10.1128/AEM.68.8.3919-3924.2002

Li L, Mei R, Klausner JF (2017) Lattice Boltzmann models for the convection-diffusion equation: D2Q5 vs D2Q9. Int J Heat Mass Transf 108:41-62.

https://doi.org/10.1016/j.ijheatmasstransfer.2016.11.092

Lin J, Xu Y, Zhang Y (2020) Simulation of linear and nonlinear advection-diffusion-reaction problems by a novel localized scheme. Appl Math Lett 99:106005. https://doi.org/10.1016/j.aml.2019.106005

Matur MG, Müller J, Kuttler C, Hense BA. 2015. An Approximative Approach for Single Cell Spatial Modeling of Quorum Sensing. Journal of Computational Biology 22:227-235. DOI: $10.1089 / \mathrm{cmb} .2014 .0198$

Peer) reviewing PDF | (2020:04:47932:1:0:NEW 11 Jul 2020) 
450

451

452

453

454

455

456

457

458

459

460

461

462

463

464

465

466

467

468

469

470

471

472

473

474

475

476

477

478

479

480

481

482

483

484

485

486

487

488

489

490

491

492

493

494

495

Meyer A, Megerle JA, Kuttler C, et al (2012) Dynamics of AHL mediated quorum sensing under flow and non-flow conditions. Phys Biol 9:. https://doi.org/10.1088/1478-3975/9/2/026007

Miller MB, Bassler BL (2001) Quorum Sensing in Bacteria. Annu Rev Microbiol 55:165-199. https://doi.org/10.1146/annurev.micro.55.1.165

Mitrophanov AY, Hadley TJ, Groisman EA (2010) Positive autoregulation shapes response timing and intensity in two-component signal transduction systems. J Mol Biol 401:671680. https://doi.org/10.1016/j.jmb.2010.06.051

Pai A, Tanouchi Y, You L (2012) Optimality and robustness in quorum sensing (QS)-mediated regulation of a costly public good enzyme. Proc Natl Acad Sci U S A 109:19810-19815. https://doi.org/10.1073/pnas.1211072109

Pérez-Velázquez J, Gölgeli M, García-Contreras R (2016) Mathematical Modelling of Bacterial Quorum Sensing: A Review. Bull Math Biol 78:1585-1639. https://doi.org/10.1007/s11538-016-0160-6

Pérez-Velázquez J, Quiñones B, Hense BA, Kuttler C (2015) A mathematical model to investigate quorum sensing regulation and its heterogeneity in Pseudomonas syringae on leaves. Ecol Complex 21:128-141. https://doi.org/10.1016/j.ecocom.2014.12.003

Redfield RJ (2002) Is quorum sensing a side effect of diffusion sensing? Trends Microbiol 10:365-370. https://doi.org/10.1016/S0966-842X(02)02400-9

Ribbe J, Maier B (2016) Density-Dependent Differentiation of Bacteria in Spatially Structured Open Systems. Biophys J. 110:1648-1660. https://doi.org/10.1016/j.bpj.2016.03.007.

Schluter J, Schoech AP, Foster KR, Mitri S (2016) The Evolution of Quorum Sensing as a Mechanism to Infer Kinship. PLoS Comput Biol 12:1-18. https://doi.org/10.1371/journal.pcbi.1004848

Solano C, Echeverz M, Lasa I (2014) Biofilm dispersion and quorum sensing. Curr Opin Microbiol 18:96-104. https://doi.org/10.1016/j.mib.2014.02.008

Stewart PS (2003) Diffusion in Biofilms. J Bacteriol 185:1485-1491. https://doi.org/10.1128/JB.185.5.1485-1491.2003

Tang L, Zhao CY, Wang XH, et al (2015) Macromolecular crowding of molecular imprinting: A facile pathway to produce drug delivery devices for zero-order sustained release. Int $\mathrm{J}$ Pharm 496:822-833. https://doi.org/10.1016/j.ijpharm.2015.10.031

Tang Y, Valocchi AJ, Werth CJ, Liu H (2013) An improved pore-scale biofilm model and comparison with a microfluidic flow cell experiment. Water Resour Res 49:8370-8382

Thullner M, Baveye P (2008) Computational pore network modeling of the influence of biofilm permeability on bioclogging in porous media. Biotechnol Bioeng 99:1337-1351. https://doi.org/10.1002/bit.21708

Trovato A, Seno F, Zanardo M, et al (2014) Quorum vs. diffusion sensing: A quantitative analysis of the relevance of absorbing or reflecting boundaries. FEMS Microbiol Lett 352:198-203. https://doi.org/10.1111/1574-6968.12394

Vaughan BL, Smith BG, Chopp DL (2010) The Influence of Fluid Flow on Modeling Quorum Sensing in Bacterial Biofilms. Bull Math Biol 72:1143-1165. https://doi.org/10.1007/s11538-009-9485-8

Ward JP, King JR, Koerber AJ, Williams P, Croft JM, Sockett RE. Mathematical modelling of quorum sensing in bacteria. Math Med Biol. 2001;18: 263-292. https://doi.org/10.1093/imammb18.3.263.

Whiteley M, Diggle SP, Greenberg EP (2017) Progress in and promise of bacterial quorum sensing research. Nature 551:313-320. https://doi.org/10.1038/nature24624 
496 Xavier JB, Foster KR (2007) Cooperation and conflict in microbial biofilms. Proc Natl Acad Sci 497 U S A 104:876-881. https://doi.org/10.1073/pnas.0607651104

498 Yates EA, Philipp B, Buckley C, et al (2002) N-Acylhomoserine Lactones Undergo Lactonolysis 499 in a pH-, Temperature-, and Acyl Chain Length-Dependent Manner during Growth of 500 Yersinia pseudotuberculosis and Pseudomonas aeruginosa. Infect Immun 70:5635-5646. $501 \quad$ https://doi.org/10.1128/IAI.70.10.5635-5646.2002

502

503 


\section{Figure 1}

Mathematical investigation of microbial quorum sensing under various flow conditions Steady state concentration fields of signaling molecules at three Peclet numbers

Concentration fields of signal concentration $(A)$ produced by microorganisms located at $x=1$ and $y=0$ with $D a=5$ and (a) $P e=1$, (b) $P e=5$, and (c) $P e=10$, without autoinduction $(F=$ $0)$. Note the difference in scale on the horizontal axis.
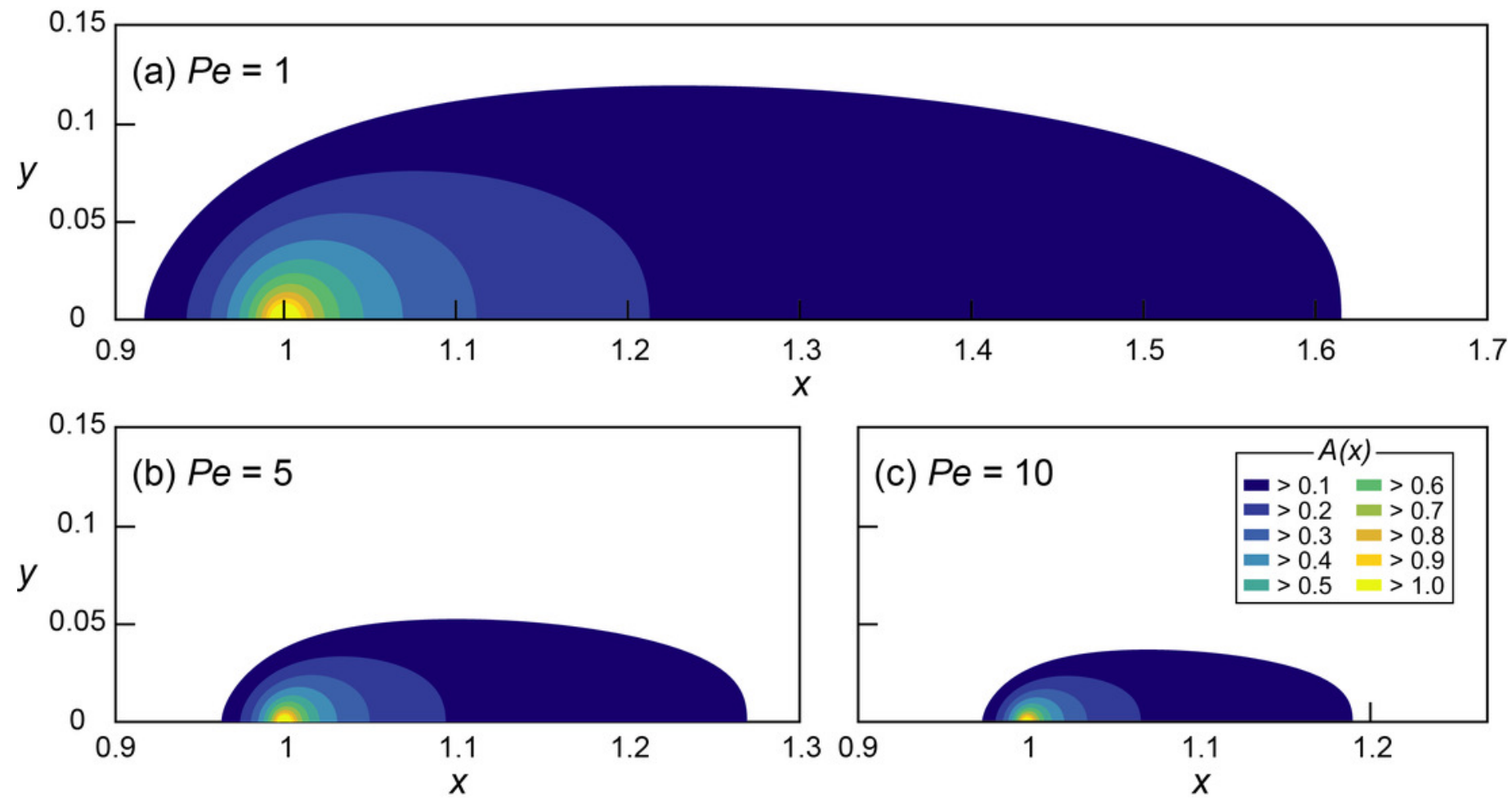
Figure 2

Threshold Damkohler numbers under a range of Peclet numbers for quorum sensing induction

The relationship between the threshold $D a$ for QS induction $\left(D a_{\theta}\right)$ and $P e$. The simulation results (block dots) were fitted using the power regression (red line; Eq. 8).

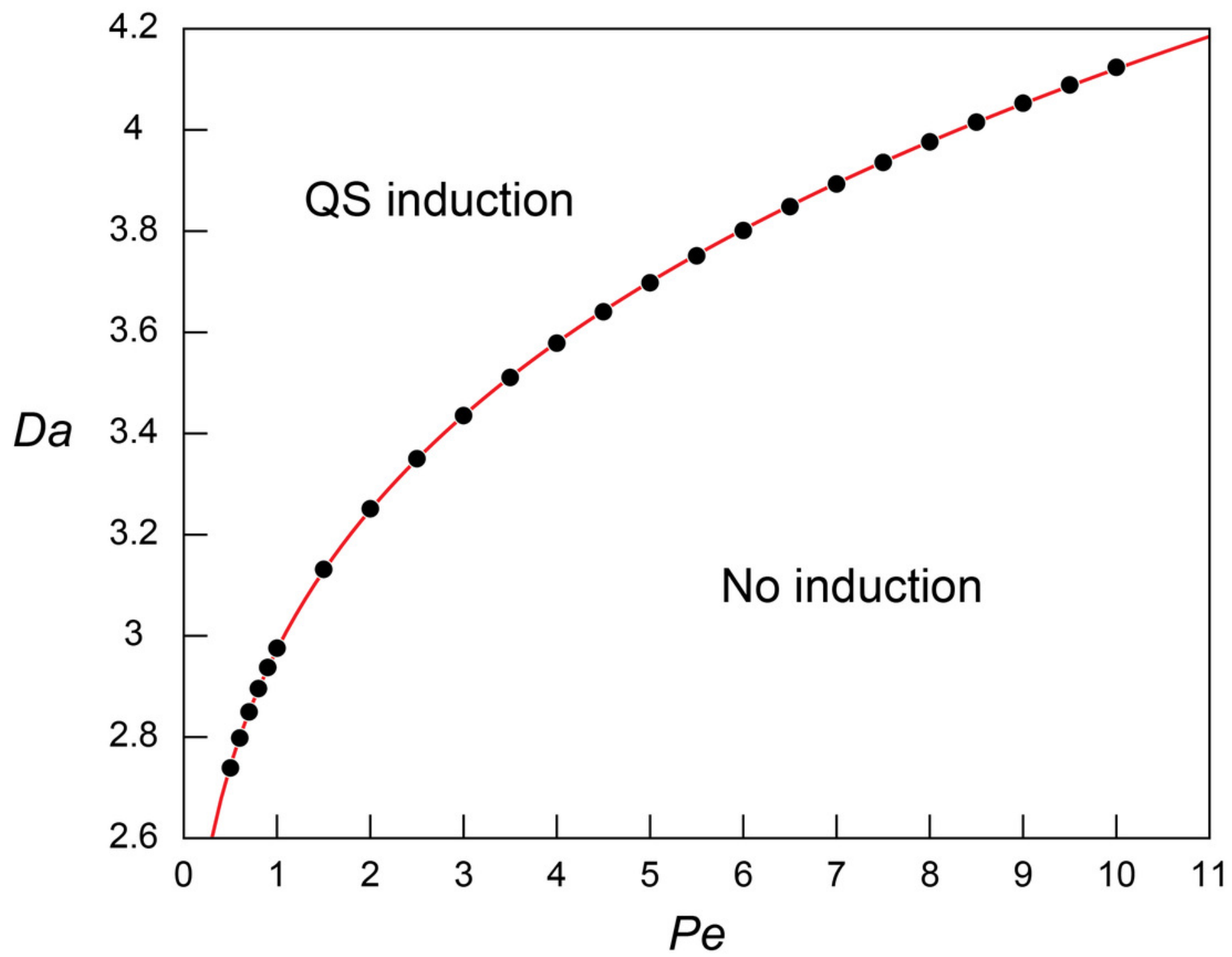




\section{Figure 3}

Constructing empirical relationships between the transport distance of signaling molecules and Peclet numbers

(a) Simulated (dots) and reconstructed (lines) concentration profiles along the bottom of the flow channel $(y=0.001)$ at $P e=1$ and $D a=D a_{\theta}=2.98$. The upstream $\left(x \leq 1 ; A_{u p}(x)\right.$; solid line) and downstream ( $x>1 ; A_{d n}(x)$; dashed line) concentration profiles were obtained from Eqs. 9 and 10, respectively. (b-d) The coefficients for $A_{u p}(x)\left(a_{u p}, b_{u p}\right.$, and $\left.c_{u p}\right)$ and $A_{d n}(x)\left(a_{d n}\right.$, $b_{d n}$, and $c_{d n}$ ) obtained from the parametric regressions of the simulated concentration profiles at each simulated Pe conditions with Eq. 9 (black dots) and Eq. 10 (white dots), respectively. The solid and dashed lines are the exponential (Eqs. 11-13) and power fits (Eqs. 14-16) of the estimated coefficients as a function of $P e$.
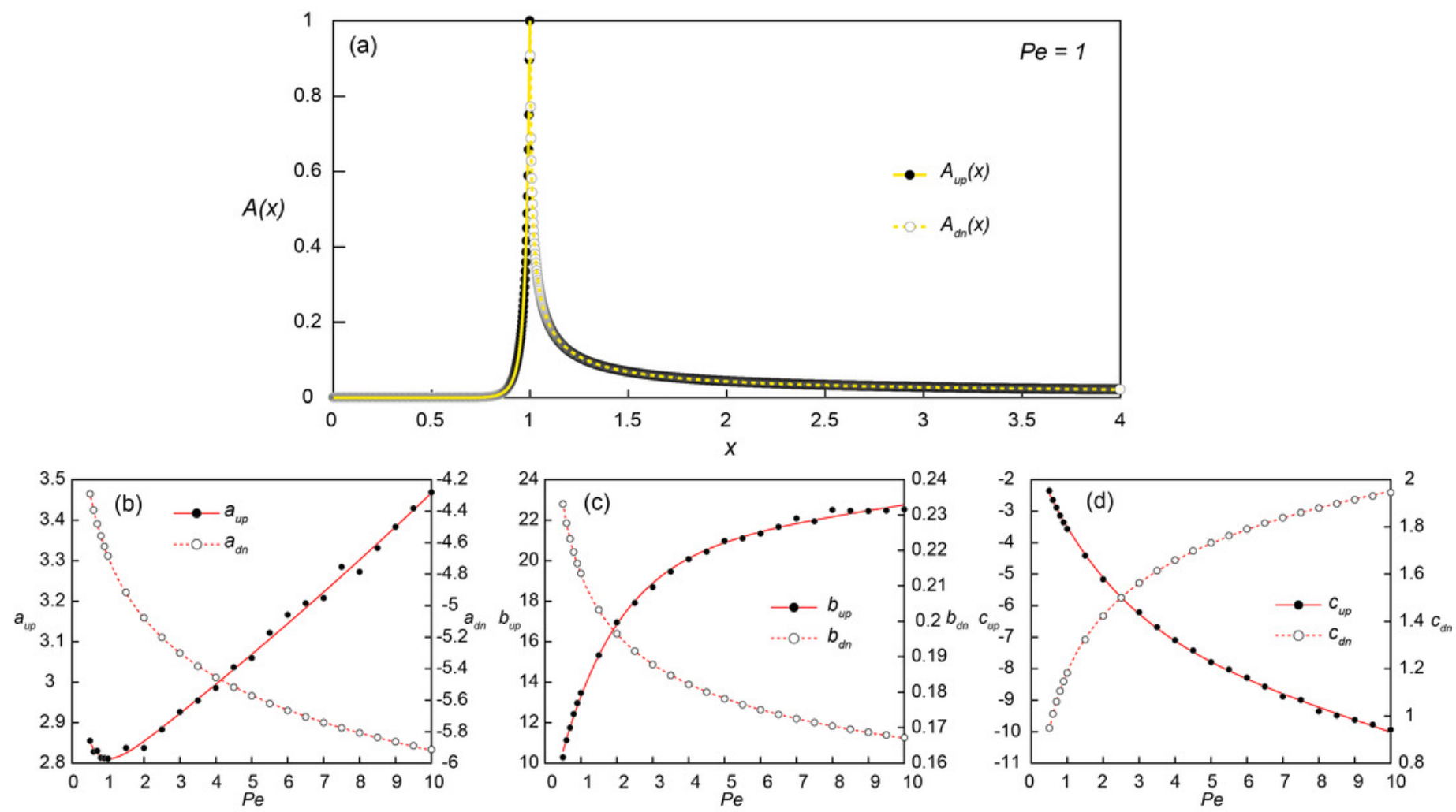


\section{Figure 4}

Transport distances of signaling molecules with and without autoinduction

Upstream $\left(d_{u p}\right)$ and downstream $\left(d_{d n}\right)$ transport distances (a) without $(F=0)$ and (b) with ( $F$ $=10$ ) enhanced signal production for the concentration ratios $\left(0.1 \leq A / A_{\max } \leq 0.9\right)$ at $P e=1$,

5 , and 10, and (c) the enlarged barplot of upstream transport distances with $F=10$. Note the different scale of the horizontal axes between panels.

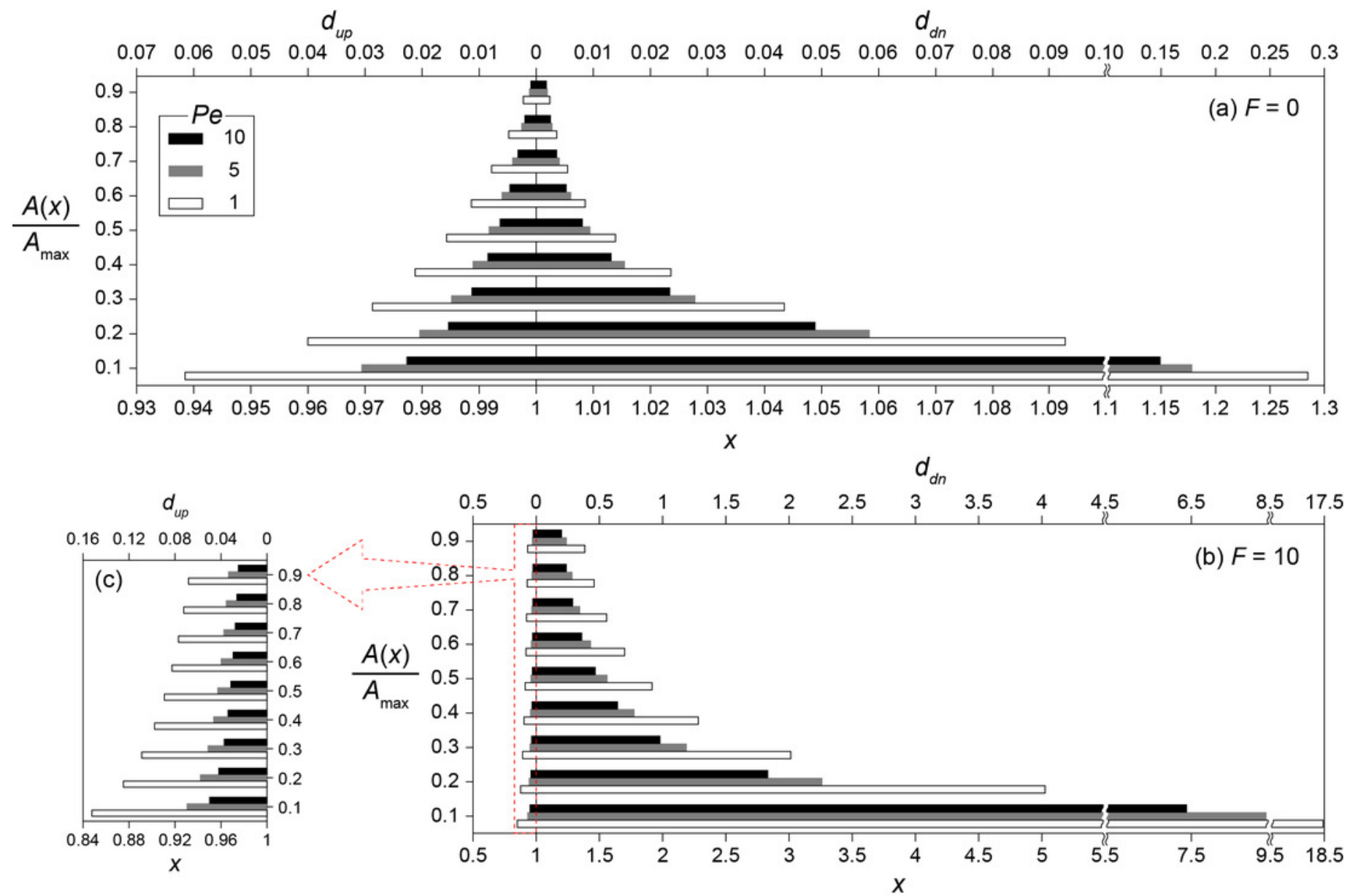


Figure 5

Quorum sensing amongst multiple microbial aggregates

Concentration profile (a) without $(F=0)$ and $(b)$ with $(F=10)$ enhanced signal production where four aggregates are located at $x_{1}=0.4, x_{2}=1, x_{3}=1.096$, and $x_{4}=1.7$. Black dots are the simulated results and the yellow lines represent the profile from Eq. 18.
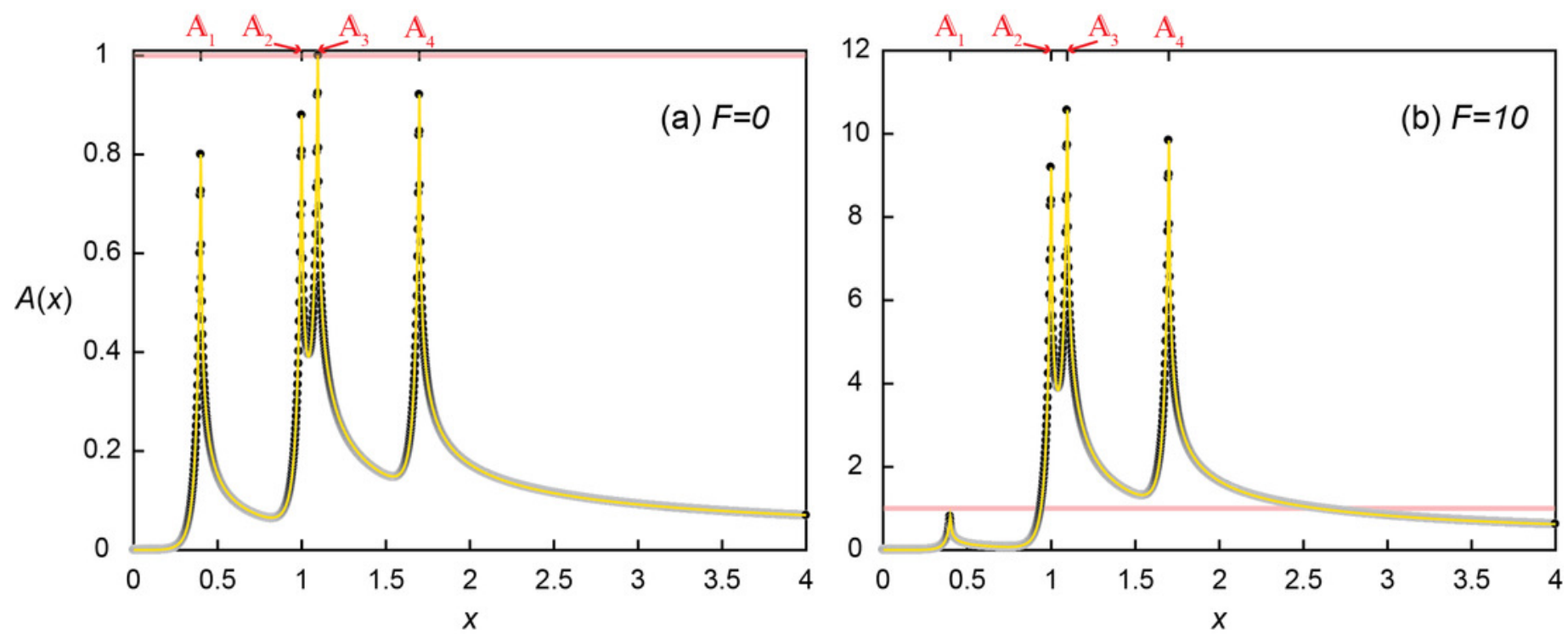